\title{
Hyperbaric oxygen therapy ameliorates pain in overweight or obese patients diagnosed with fibromyalgia
}

\author{
JUAN-GUILLERMO WINFIELD-VARGAS ${ }^{1}$, JULIO C. RAMÍREZ-NAVA ${ }^{2}$, VALENTÍN RODRÍGUEZ-AYALA ${ }^{2}$, \\ JUAN A. CRUZ-SÁNCHEZ ${ }^{3}$, HÉCTOR SOLÍS-CHAGOYÁN ${ }^{4}$, ARNOLDO AQUINO-GÁLVEZ ${ }^{5}$, \\ BIANCA S. ROMERO-MARTÍNEZ ${ }^{6}$, LUIS M. MONTAÑO ${ }^{6}$ and EDGAR FLORES-SOTO ${ }^{6}$ \\ ${ }^{1}$ Posgraduate School of Naval Health, Naval University, Secretary of The Navy of Mexico, 04800 Veracruz; \\ ${ }^{2}$ Hospital Specialties Naval Veracruz, Specialist in Hyperbaric and Underwater Medicine; \\ ${ }^{3}$ Department of Rheumatology, Specialties Naval Hospital of Veracruz, 91700 Veracruz; ${ }^{4}$ Laboratory of Neuropharmacology, \\ National Institute of Psychiatry Ramon de la Fuente Muñiz, 14370 CDMX; ${ }^{5}$ Laboratory of Molecular Biology, \\ National Institute of Respiratory Diseases 'Ismael Cosio Villegas', 14080 CDMX; \\ ${ }^{6}$ Departament of Pharmacology, Faculty of Medicine, National Autonomous University of Mexico, 04510 CDMX, Mexico
}

Received February 9, 2021; Accepted May 10, 2021

DOI: 10.3892/wasj.2021.107

\begin{abstract}
Fibromyalgia is a syndrome characterized by widespread musculoskeletal pain, fatigue, poor sleep quality, allodynia and paresthesia. Therapy for this syndrome consists of pharmacological and non-pharmacological approaches focused on controlling pain and fatigue to improve the quality of life of those affected. The present study aimed to determine whether hyperbaric oxygen therapy (HBOT) can be considered a useful adjuvant of pre-existing pharmacological treatment to reduce pain and improve the quality of life of patients with fibromyalgia. Patients diagnosed with fibromyalgia were enrolled in the present study following a clinical evaluation. All participants received 20 sessions of HBOT (five sessions/week, Monday-Friday). The sessions consisted of breathing for $60 \mathrm{~min}$ with $100 \%$ oxygen at a 2.0 atmospheric absolute pressure. Pain, fatigue, quality of life and the number of drugs used were measured to assess the effectiveness of HBOT. Prior to HBOT, the patients presented with an average visual analog scale score of $8.06 \pm 0.475$, which was reduced to $3.86 \pm 0.748$ following treatment. In addition, the functional assessment of chronic illness therapy-fatigue score indicated amelioration of this symptom by HBOT, reducing the mean score from $44.86 \pm 2.19$ to $21.6 \pm 1.09$. Furthermore, the quantification of the quality of life using the fibromyalgia impact questionnaire reduced the score from $76.66 \pm 4.11$ to $47.6 \pm 2.67$.
\end{abstract}

Correspondence to: Dr Edgar Flores-Soto, Department of Pharmacology, Faculty of Medicine, National Autonomous University of Mexico, 04510 CDMX, Mexico

E-mail: edgarfloressoto@yahoo.com.mx

Key words: fibromyalgia, fibromyalgia impact questionnaire, hyperbaric oxygen therapy, obesity, overweight, pain, visual analogue scale
As regards the number of drugs administered to the patients, this decreased from $3.13 \pm 0.764$ to $1.53 \pm 0.85$. Taken together, the results of the present study suggest that HBOT may be used as an effective adjuvant in the treatment of fibromyalgia, reducing both pain and fatigue. In addition, this treatment improves the quality of life of those affected and may be used to avoid polypharmacy.

\section{Introduction}

Fibromyalgia is a multifactorial syndrome characterized by widespread musculoskeletal pain, fatigue, sleep disturbances, morning stiffness, loss of functional capacity, mood alterations, as well as a decline in memory, cognition, headaches and a myriad of other visceral and cognitive maladies $(1,2)$. These symptoms negatively affect the quality of life of patients. Fibromyalgia is diagnosed using the criteria proposed by the American College of Rheumatology. In 2016, the latest revision of these criteria re-established the importance of generalized pain as the main symptom (3), but also considered other symptoms equally as important for the diagnosis considering these criteria. The estimated global population affected by this disease ranges between $0.5-5.8 \%$ (4), and the disease particularly affects women (2.4-6.8\%) (5). Fibromyalgia is a disabling syndrome with no effective pharmacological cure; thus, recent alternative therapeutic strategies have been proposed to mitigate pain and fatigue, and avoid the side-effects of polypharmacy (6).

Chronic musculoskeletal pain has a worldwide prevalence of $30 \%$ (4). Managing the unpleasant sensory and emotional experience associated with tissue damage by patients with chronic pain is a difficult predicament that requires a multidisciplinary approach, particularly in patients suffering from fibromyalgia, in whom the control system that interprets and distinguishes painful stimuli is overly sensitive (7). Its treatment is based on the combination of pharmacological and non-pharmacological approaches and interventional 
techniques, which usually only achieve modest results in pain relief; in this regard, the absence of positive results or the generation of adverse effects can be the reason to stop treatment (7). Furthermore, pain and fatigue are frequently associated with comorbidities, such as sleep disturbances, depression and metabolic syndrome, which render treatment difficult (7). Obesity is an increasingly prevalent health concern in the Mexican population; thus, the risk of comorbidities associated with metabolic deficiencies and fibromyalgia can increase progressively. In this regard, the amelioration of pain and fatigue by an effective therapy can allow the opportunity for obese patients with fibromyalgia to lose weight by controlling physical activity to avoid chronic comorbidities. The greater the physical and mental deterioration caused by comorbidities, the more difficult it is to identify effective personalized treatment $(7,8)$.

Among non-pharmacological alternatives for the treatment of fibromyalgia, hyperbaric oxygen therapy (HBOT) has been proposed as a novel effective therapy; however, further studies are required in populations with different genetic, ethnic and cultural traits to verify its effectiveness (8-10). Although HBOT was initially designed to treat diving-related injuries, promising results have been observed as a nociceptive, inflammatory and neuropathic pain analgesic (7). HBOT is a treatment providing $100 \%$ oxygen at a pressure above one atmosphere absolute (ATA). The main mechanisms of action activated by HBOT to reduce pain can be reversed by hypoxia in dysfunctional tissues by increasing the partial pressure of arterial oxygen (7-10). In addition, it can exert hydrostatic pressure to stimulate elimination of inert gas from tissues (11).

The present study aimed to determine whether HBOT is effective in treating pain and fatigue, as well as improving the quality of life and reducing the number of drugs used by patients diagnosed with fibromyalgia in a Mexican population.

\section{Materials and methods}

Patients. A total of 15 patients diagnosed with fibromyalgia who had received medical care at the Department of Rheumatology, Naval Specialty Hospital (Veracruz, Mexico) March, 2019 to December, 2019, were referred to the Hyperbaric Medicine Service to determine the efficacy of HBOT to reduce pain and improve their quality of life. The present study was approved by the Institutional Bioethics Committee of Hospital Naval de Alta Especialidad de Veracruz and performed in accordance with the Declaration of Helsinki. Written informed consent was provided by all participants prior to the study start.

Beneficiaries of health services at the Naval Specialty Hospital are active or retired naval service members. Patients diagnosed with fibromyalgia, regardless of sex or age, were initially accepted; however, a complete clinical evaluation was performed before candidates underwent HBOT. The exclusion criteria were as follows: i) Patients with claustrophobia; ii) proliferative diabetic retinopathy; iii) history of oncological pathology; iv) pregnant women and v) patients with pneumothorax disease. Patients were eliminated from the study if they decided to leave or discontinue treatment, as well as those who missed $>5$ sessions or did not survive.

The treatment comprised 20 sessions of HBOT (five sessions/week, Monday-Friday), breathing $60 \mathrm{~min}$ of
$100 \%$ oxygen at 2.0 ATA. A total of 20 sessions were selected based on previous results of HBOT, from the 15th-40th sessions (8-10). Before the first session, as well as at the end of treatment, the following parameters were assessed: Visual analog scale (VAS) score to detect changes in pain; A VAS is usually a $10-\mathrm{cm}$ horizontal line, delimiting extremes with perpendicular lines; one extreme is defined by verbal expressions, such as 'no pain' and accompanied by the number ' 0 '; the other extreme is mentioned as 'maximum pain ever experienced' and accompanied by the number '10' (12), and functional assessment of chronic illness therapy-fatigue (FACIT-F); The 13-item FACIT-F is a test used to evaluate the impact of fatigue on different aspects of the patients functioning, such as physical, functional, emotional, and social over the past seven days. Items are measured on a four-point Likert scale from 0 (not at all) to 4 (very much). Scores are added together and the higher the result, the greater the impact of fatigue on aspects of the patients' lives (13). In addition, the quality of life of patients was evaluated using the fibromyalgia impact questionnaire (FIQ) at the beginning and end of treatment; the FIQ scale ranges from 0-100, whereby 0 represents the best quality of life and 100 represents the worst quality of life (9). The number of drugs patients used for pain relief was also recorded.

Statistical analysis. All data were analyzed by GraphPad Prism ${ }^{\circledR}$ version 3.10 (GraphPad Software, Inc.). A paired Student's t-test was used to compare differences before and after HBOT (20 sessions). Data are presented as the mean \pm standard error of the mean. $\mathrm{P}<0.05$ was considered to indicate a statistically significant difference.

\section{Results}

Sociodemographic data of patients with fibromyalgia. To the best of our knowledge, no previous studies to date in Mexico have investigated the efficacy of HBOT to control and mitigate the pain, and improve the quality of life of patients with fibromyalgia.

The present study was performed at the Naval Specialty Hospital (Veracruz, Mexico), which included 15 women (mean age, $50.53 \pm 3.77$ years; age range, $32-72$ years; $67 \%$ were $\leq 55$ years; Fig. 1) who met the selection criteria to receive 20 sessions of HBOT at 2 ATA for $60 \mathrm{~min}$. A total of eight patients had a Bachelor's degree, while seven had graduated from high school or middle school. A total of eight patients were housewives, while seven were active professionals. A total of 10 patients had primary fibromyalgia and five had secondary fibromyalgia. The average body mass index (BMI) value was 29.26 \pm 0.54 ; six patients were classified as obese $\left(\mathrm{BMI} \geq 30 \mathrm{~kg} / \mathrm{m}^{2}\right)$ and nine were overweight (BMI $\geq 25$ and $<30 \mathrm{~kg} / \mathrm{m}^{2}$ ).

Evaluating the effectiveness of HBOT as a therapeutic adjuvant for the treatment of fibromyalgia. To determine whether HBOT may be used as an alternative adjuvant in the pharmacological treatment of patients with fibromyalgia, two indicators of symptoms were quantified, and two indicators were measured to determine decline or improvement of quality of life. As regards the former, pain and 


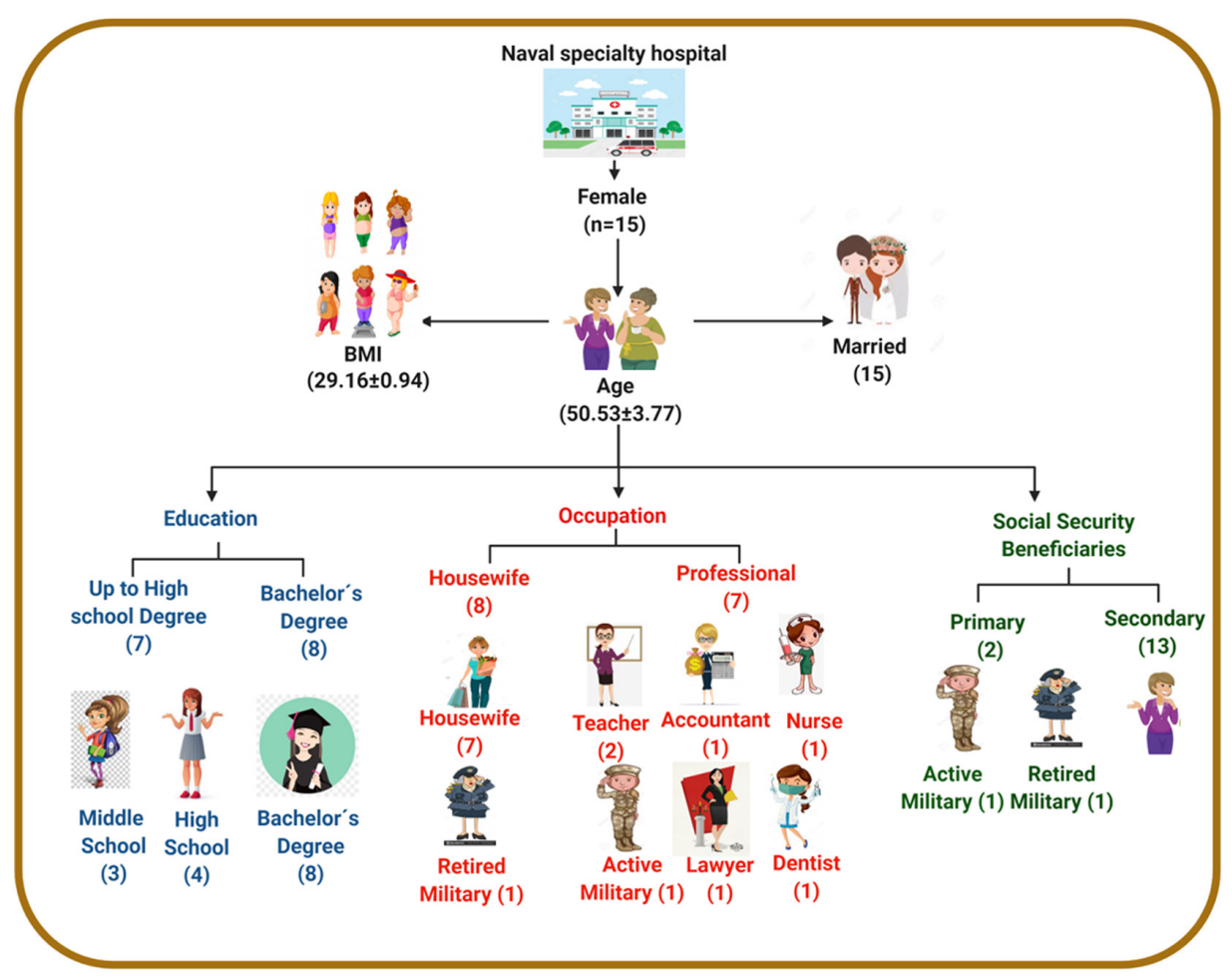

Figure 1. Sociodemographic data of patients diagnosed with fibromyalgia. BMI, body mass index.

fatigue intensity were evaluated using reliable and validated questionnaires.

Pain evaluation involved the quantification of the VAS score, which is considered an efficient indicator for pain intensity and is used to differentiate between patients with fibromyalgia and healthy individuals. Prior to the HBOT sessions, patients had an average score of $8.06 \pm 0.475$, which corresponded to a high level of pain. Following HBOT, perceived pain was remeasured and the mean VAS score was $3.86 \pm 0.748$, which indicated a low level of pain (Fig. 2A and Table I). Notably, $73 \%$ of patients had an initial VAS score of 8 or 9 and $27 \%$ of patients had a score of 7 ; whereas at the end of treatment, $64 \%$ of patients had a score $\leq 4$ and the remaining $36 \%$ had a score of 5 or 6 (Table II). Taken together, these results suggest that breathing $100 \%$ oxygen at 2 ATA is an adequate therapeutic procedure to mitigate perceived pain in fibromyalgia.

Fatigue is an important symptom that contributes to the decline of the quality of life of patients with fibromyalgia $(5,6)$. This symptom was evaluated using the FACIT-F questionnaire of 13 factors, which were scored as follows: 0, no fatigue; 1, minimal fatigue; 2 , some; 3 , quite a bit; and 4 , significant fatigue. The mean initial score was $44.86 \pm 2.19$ (Fig. 2B and Table I); $93 \%$ of the patients $(n=14)$ accumulated a score of $\geq 40$ between 40 and 52 , representing a considerable level of fatigue; only one patient had a score of 39 (Table II). However, the mean final score was $21.6 \pm 1.09$ (Fig. $2 \mathrm{~B}$ and Table I); the score of $80 \%$ of patients decreased to $\leq 22$, representing a level of tolerable fatigue and $20 \%$ had a score $\geq 26$ (between 26 and 31) (Table II). Collectively, these results suggest that fatigue significantly decreases with HBOT.

The FIQ was used to evaluate the decline or improvement in quality of life, which included determining the number
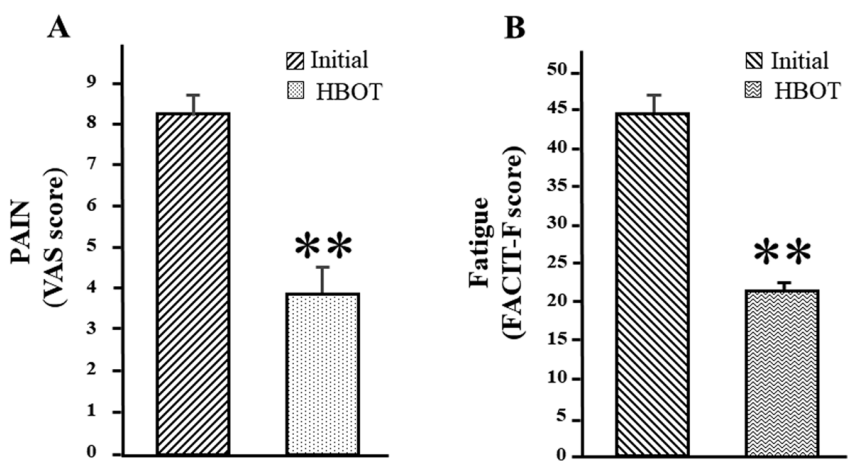

Figure 2. Pain and fatigue evaluation in patients with fibromyalgia. (A) Pain was evaluated using the VAS score, while (B) fatigue was evaluated using the FACIT-F score. A paired Student's t-test was used to compare differences between two groups $(n=15)$. Data was presented as the mean \pm standard error of the mean. ${ }^{* *} \mathrm{P}<0.01$ vs. initial stage, before treatment. VAS, visual analog scale; FACIT-F, functional assessment of chronic illness therapy-fatigue.

of drugs used by patients. Fibromyalgia is a multifactorial syndrome that markedly affects the quality of life of patients. The FIQ scale ranges from 0-100, whereby 0 represents the best quality of life and 100 represents the worst quality of life (9). In the present study, the FIQ score significantly decreased from $76.66 \pm 4.11$ at the beginning of the study to $47.6 \pm 2.67$ following HBOT (Fig. 3A and Table I). Taken together, these results suggest that $\mathrm{HBOT}$ is effective in improving the quality of life of patients with fibromyalgia.

Fibromyalgia is an unknown-etiological syndrome, without a specific pharmacological treatment. The drugs used to manage this disease aim to reduce the incapacitating symptoms (1). If a certain improvement in symptoms is made by the patients, the side effects limits the use of several drugs 
Table I. Results of VAS, FACIT, FIQ and drug scores of patients before and after HBOT $(\mathrm{n}=15)$.

\begin{tabular}{lcccc}
\hline Before/after treatment & VAS $($ mean \pm SEM) & FACIT-F (mean \pm SEM) & FIQ $($ mean \pm SEM) & Drugs $($ mean \pm SEM) \\
\hline Initial (before treatment) & $8.06 \pm 0.475$ & $44.86 \pm 2.19$ & $76.66 \pm 4.11$ & $3.13 \pm 0.764$ \\
HBOT & $3.86 \pm 0.748^{\mathrm{a}}$ & $21.6 \pm 1.09^{\mathrm{a}}$ & $47.6 \pm 2.67^{\mathrm{a}}$ & $1.53 \pm 0.85^{\mathrm{a}}$ \\
\hline
\end{tabular}

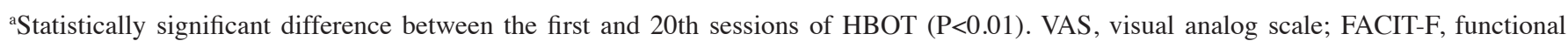
assessment of chronic illness therapy-fatigue; FIQ, fibromyalgia impact questionnaire, HBOT, hyperbaric oxygen therapy.

Table II. VAS and FACIT scores of patients before and after HBOT $(n=15)$.

\begin{tabular}{|c|c|c|c|c|}
\hline \multirow{2}{*}{$\frac{\text { Before/after treatment }}{\text { Initial (before treatment) }}$} & \multicolumn{2}{|c|}{ VAS ( $\%$ of patients with each score) } & \multicolumn{2}{|c|}{ FACIT ( $\%$ of patients with each score) } \\
\hline & Score $8-9$ & Score 7 & Score $\geq 40$ & Score $\leq 39$ \\
\hline & 73 & 27 & 93 & 7 \\
\hline \multirow[t]{2}{*}{ HBOT } & Score 2-4 & Score 5-6 & Score $\leq 22$ & Score $\geq 26$ \\
\hline & 64 & 36 & 80 & 20 \\
\hline
\end{tabular}

VAS, visual analog scale; FACIT-F, functional assessment of chronic illness therapy-fatigue.

A

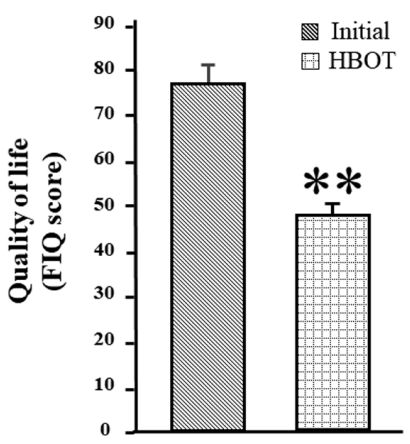

B

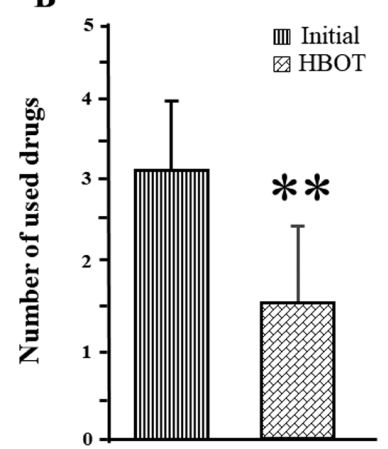

Figure 3. Evaluation of quality of life of patients with fibromyalgia and recording the number of drugs used. (A) Quality of life was assessed according to FIQ. (B) The number of used drugs was plotted. A paired Student's t-test was used to compare differences between two groups. ${ }^{* *} \mathrm{P}<0.01$, vs. initial stage, before treatment. FIQ, fibromyalgia impact questionnaire.

simultaneously. Hence, a positive impact of treatment is indicated by the reduction in the number of drugs used to mitigate the pain or the fatigue in patients. The drug number was evaluated at the beginning and end of treatment. The average number of initial drugs used was $3.13 \pm 0.764$, which decreased to $1.53 \pm 0.85$ drugs following HBOT (Fig. $3 \mathrm{~B}$ and Table I). Collectively, these results suggest that HBOT may be a promising alternative to reduce the use of drugs with side effects to mitigate pain and fatigue and improve the quality of life of patients with fibromyalgia.

\section{Discussion}

The patient population participating in the present study was predominantly married active professional women, $<55$ years of age, whose BMI corresponded to being overweight or obese. One fifth of the patients were diagnosed with secondary

fibromyalgia, which implies comorbidities, such as stroke, mood disorders or diabetes (10). The baseline perception in this subgroup comprised high levels of pain intensity (VAS scores between 8 and 9) and fatigue (FATIC-F between 42-52), suggesting that these women cope with severe levels of pain and fatigue daily $(10,14)$. Previous studies have reported a close association between chronic pain intensity or fatigue and high BMI (14-17), which may explain the results of the present study.

The effectiveness of HBOT to treat Mexican women with fibromyalgia, who were overweight or obese, was evaluated in the present study by measuring perceived pain, fatigue, quality of life, as well as the number of drugs used. A previous study reported that a 50-year-old Turkish woman, with a mean BMI of $29 \mathrm{~kg} / \mathrm{m}^{2}$, had VAS and FIQ scores similar to those reported in the present study ( 8 and 73 , respectively) (18). Neural therapy consisting of intracutaneous $1 \%$ lidocaine administered as a local anesthetic into painful points was used to treat fibromyalgia in this study, and the posttreatment scores were 3.7 (VAS) and 40 (FIQ) (18). In another study, Korean women (mean age, 47 years) with a VAS score of 8.8 and FIQ score of 80 were treated with transcranial direct current stimulation; the treatment reversed perceived pain to 6 (VAS score) and decreased the FIQ score to 70 (19). Notably, no significant differences in VAS or FIQ scores were observed in Spanish women (mean age, 53 years) treated with low-intensity physical activity programs for 12 weeks (20); however, a moderate-intense aerobic exercise program effectively reduced pain to 5.7 and the FIQ score to 55 (21). In Israel, a group of women (mean age, 50 years), with an average BMI of 27, were treated with HBOT (22). In addition, Turkish women (mean age, 40 years) also treated with 15 sessions of HBOT exhibited reduced VAS scores, from 64 to 31 . Collectively, these reports suggest that non-pharmacological approaches are effective in reducing the severity of fibromyalgia symptoms.

Health must be perceived as a daily mutual commitment by subjects and institutions to favor physical, psycho-emotional 
and social wellbeing. Patients must be mindful of their eating habits and fibromyalgia treatment needs to be holistic. Thus, therapeutic management should be characterized by integrated and multidisciplinary interventions that ameliorate physical complications but also address the psychological and social aspects of the disease (23). Pain is one of the main inhibitors of patients' adherence to controlled physical activity programs (24). In addition, activity is necessary to lose weight, as being overweight or obese increases the risk of severe pain. A variety of drugs, such as muscle relaxants, analgesics, anti-inflammatories, antidepressants and anticonvulsants have been widely used as pharmacological therapy for fibromyalgia (25). A common side-effect of polypharmacy is the development of metabolic or mental health issues, aggravating patients' conditions and severely impairing their quality of life (23). Thus, HBOT may be a suitable approach to dissociate the negative feedback loop between high BMI-pain-sedentarism to implement a multidisciplinary management program to treat overweight or obese patients with fibromyalgia.

In conclusion, HBOT in patients with fibromyalgia significantly improves pain and fatigue management, as well as other aspects of the disease, such as the quality of life and the possibility to avoid the side-effects of polypharmacy. However, further studies are required to establish a standardized treatment scheme with HBOT, not only in fibromyalgia, but also in other chronic disorders in which widespread pain is a symptom that generates inability. Taken together, the results of the present study support the hypothesis that this procedure can be used as an effective adjuvant to contribute to the wellbeing of female patients that suffer from fibromyalgia. Reduction of pain and fatigue in overweight or obese patients opens the opportunity to initiate an increment in physical activity and exercise to lose weight and decrease the risk of health-associated chronic degenerative comorbidities.

\section{Acknowledgements}

The publication of the present study is required by JGWV to obtain the title of Specialist in Underwater and Hyperbaric Medicine in the Universidad Naval, Escuela de Posgrados en Sanidad Naval, Secretaría de Marina Armada de México to whom he is grateful for the instructions received during his studies.

\section{Funding}

No funding was received.

\section{Availability of data and materials}

The datasets used and/or analyzed during the current study are available from the corresponding author on reasonable request.

\section{Authors' contributions}

JGWV analyzed and interpreted the patient data. JCRN, VRA and JACS recruited patients and performed the hyperbaric oxygen therapy. HSC, LMM, EFS, AAG and BSRM analyzed and interpreted the patient data. HSC, LMM and EFS were major contributors in the writing of the manuscript. JGWV and EFS confirm the authenticity of all the raw data. All authors have read and approved the final manuscript.

\section{Ethics approval and consent to participate}

The present study was approved by the institutional Ethics Committee of Hospital Naval de Alta Especialidad de Veracruz, and the committee's reference is 83/19. Informed consent for participation in the study was obtained from all participants.

\section{Patient consent for publication}

Not applicable.

\section{Competing interests}

The authors declare that they have no competing interests.

\section{References}

1. Sumpton JE and Moulin DE: Fibromyalgia. Handb Clin Neurol 119: 513-527, 2014.

2. López-Pousa S, Garre-Olmo J, de Gracia M, Ribot J, Calvó-Perxas L and Vilalta-Franch J: Development of a multidimensional measure of fibromyalgia symptomatology: The comprehensive rating scale for fibromyalgia symptomatology. J Psychosom Res 74: 384-392, 2013.

3. Wolfe F, Clauw DJ, Fitzcharles MA, Goldenberg DL, Häuser W, Katz RL, Mease PJ, Russell AS, Russell IJ and Walitt B: 2016 revisions to the 2010/2011 fibromyalgia diagnostic criteria. Semin Arthritis Rheum 46: 319-329, 2016.

4. Clauw DJ: Fibromyalgia and related conditions. Mayo Clin Proc 90: 680-692, 2015.

5. Gómez-de-Regil L and Estrella-Castillo DF: Psychotherapy for physical pain in patients with fibromyalgia: A systematic review. Pain Res Manag 2020: 3408052, 2020.

6. Maffei ME: Fibromyalgia: Recent advances in diagnosis, classification, pharmacotherapy and alternative remedies. Int J Mol Sci 21: 7877, 2020

7. Sutherland AM, Clarke HA, Katz J and Katznelson R: Hyperbaric oxygen therapy: A new treatment for chronic pain? Pain Pract 16: 620-628, 2016

8. Yildiz S, Kiralp MZ, Akin A, Keskin I, Ay H, Dursun H and Cimsit M: A new treatment modality for fibromyalgia syndrome: Hyperbaric oxygen therapy. J Int Med Res 32: 263-267, 2004.

9. Atzeni F, Casale R, Alciati A, Masala IF, Batticciotto A, Talotta R, Gerradi MC, Salafi F and Sarzini-Puttini P: Hyperbaric oxygen treatment of fibromyalgia: A prospective observational clinical study. Clin Exp Rheumatol 37 (Suppl 116): S63-S69, 2019.

10. Lev M and Goldner L: Work volition in women with fibromyalgia: A phenomenological analysis. Disabil Rehabil: Oct 12, 2020 (Epub ahead of print). doi: 10.1080/09638288.2020.18270 50.

11. Izquierdo-Alventosa R, Inglés M, Cortés-Amador S, GimenoMallench L, Sempere-Rubio N, Chirivella J and Serra-Añó P: Comparative study of the effectiveness of a low-pressure hyperbaric oxygen treatment and physical exercise in women with fibromyalgia: Randomized clinical trial. Ther Adv Musculoskelet Dis: Jun 24, 2020 (Epub ahead of print). doi: 10.1177/1759720X20930493

12. Maurel S, Rodero B, Lopez-del-Hoyo Y, Luciano JV, Andrés E, Roca M, Bergos Rdel M, Ruiz-Lancina Y and García-Campayo J: Correlational analysis and predictive validity of psychological constructs related with pain in fibromyalgia. BMC Musculoskelet Disord 12: 4, 2011.

13. Webster K, Cella D and Yost K: The functional assessment of chronic Illness therapy (FACIT) measurement system: Properties, applications, and interpretation. Health Qual Life Outcomes 1: 79, 2003.

14. Bennett $\mathrm{MH}$ and Mitchell SJ: Emerging indications for hyperbaric oxygen. Curr Opin Anaesthesiol 32: 792-798, 2019. 
15. Lorente-Ceron L, Garcia-Ríos MC, Navarro-Ledesma S, Tapia-Haro RM, Casas-Barragan A, Correa-Rodriguez M and Aguilar Ferrandiz ME: Functional status and body mass index in postmenopausal women with fibromyalgia: A case-control study. Int J Environ Res Public Health 16: 4540, 2019.

16. Gavilán-Carrera B, Acosta-Manzano P, Soriano-Maldonado A, Borges-Cosic M, Aparicio VA, Delgado-Fernandez $M$ and Segura-Jimenez V: Sedentary time, physical activity, and sleep duration: Associations with body composition in fibromyalgia. The Al-andalus project. J Clin Med 8: 1260, 2019. x

17. Correa-Rodríguez M, Mansouri-Yachou JE, Casas-Barragán A, Molina F, Rueda-Medina B and Aguilar-Ferrandiz ME: The association of body mass index and body composition with pain, disease activity, fatigue, sleep and anxiety in women with fibromyalgia. Nutrients 11: 1193, 2019.

18. Balevi Batur E and Atan T: Neural therapy for fibromyalgia: Myth or improving quality of life? Int J Clin Pract 21: 13719, 2020.

19. Kang JH, Choi SE, Park DJ, Xu H, Lee JK and Lee SS: Effects of add-on transcranial direct current stimulation on pain in Korean patients with fibromyalgia. Sci Rep 10: 12114, 2020.

20. Sauch-Valmaña G, Vidal-Alaball J, Roura-Poch P, Mendiroz-Peña J, Panades-Zafra R, Cantero-Gomez FX, Ruiz-Comellas A and Barranco-Reixachs D: Effects of a physical exercise program on patients affected with fibromyalgia. J Prim Care Community Health 11: 2150132720965071, 2020.
21. Gómez-Hernández M,Gallego-IzquierdoT,Martínez-MerineroP, Pecos-Martín D, Ferragut-Garcías A, Hita-Contreras F, Martínez-Amat A, Montañez-Aguilera FJ and AchalandabasoOchoa A: Benefits of adding stretching to a moderate-intensity aerobic exercise programme in women with fibromyalgia: A randomized controlled trial. Clin Rehabil 34: 242-251, 2020.

22. Efrati S, Golan H, Bechor Y, Faran Y, Daphna-Tekoah S, Sekler G, Fishlev G, Ablin JN, Bergan J, Volkov O, et al: Hyperbaric oxygen therapy can diminish fibromyalgia syndrome-Prospective clinical trial. PLoS One 10: e0127012, 2015.

23. Sarzi-Puttini P, Giorgi V, Marotto D and Atzeni F: Fibromyalgia: An update on clinical characteristics, aetiopathogenesis and treatment. Nat Rev Rheumatol 16: 645-660, 2020.

24. Catala P, Lopez-Roig S, Ecija C, Suso-Ribera C and Peñacoba Puente C: Why do some people with severe chronic pain adhere to walking prescriptions whilst others won't? A cross-sectional study exploring clinical and psychosocial predictors in women with fibromyalgia. Rheumatol Int: Oct 13 , 2020 doi: 10.1007/s00296-020-04719-w [Epub ahead of print].

25. Galvão-Moreira LV, Oliveria de Castro L, Rey-Moura EC, Barros de Olivera CM, Nogueira-Neto J, Rodrigues de Sousa Gomes LV and da Cunha-Leal P: Pool-based exercise for amelioration of pain in adults with fibromyalgia syndrome: A systematic review and meta-analysis. Mod Rheumatol: Feb 25, 2021 (Epub ahead of print). doi: 10.1080/14397595.2020.1829339.

(i) $(9)$ This work is licensed under a Creative Commons Attribution-NonCommercial-NoDerivatives 4.0 International (CC BY-NC-ND 4.0) License. 